\title{
"Señora de las cuatro décadas": un estudio sobre el vínculo entre música, mujeres y edad ${ }^{1}$
}

\section{Carolina Spataro}

\section{Introducción}

\section{Resumen}

El objetivo del trabajo es indagar el papel de la música en la configuración de feminidades contemporáneas y, específicamente, estudiar los clivajes etarios y generacionales que informan dicho vínculo. En primer lugar realizaremos un recorrido sobre tres paradigmas que informan los estudios sobre música y sociedad: resonancia estructural, interpelación e identidad narrativa. A partir de allí relocalizaremos la discusión: las personas se apropian de la música de modos en que ésta se constituye en un dispositivo habilitante y promotor de la acción (DeNora, 2000).

En segundo lugar, analizaremos el relato de dos mujeres que forman parte un club de fans de Ricardo Arjona en la Argentina, un cantante y compositor guatemalteco de gran éxito hace más de una década en la industria discográfica hispanohablante. Concluiremos que la música es un recurso estético de la agencia que permite erosionar expectativas etarias y de género existentes para mujeres de mediana edad.

\section{Palabras clave}

Música. Mujeres. Edad. Generación

Carolina Spataro | carolinaspataro@yahoo.com.ar Doctora en Ciencias Sociales de la Universidad de Buenos Aires (UBA). Docente de la Carrera de Ciencias de la Comunicación de la Facultad de Ciencias Sociales (FSOC) de la UBA. Becaria posdoctoral del Consejo Nacional de Investigaciones Científicas y Técnicas (CONICET).
Mi marido me dice: "vos te casaste para ser libre". En mi época para salir tenías que pedir permiso, todo muy estructurado, no me dejaron ir al viaje de egresados, soy muy... estaba muy contenida. Y a lo mejor todo eso me llamó la atención precisamente por eso, porque ví cosas distintas, su forma de expresarse, las letras (Ana, 51 años).

Desde hace dieciséis años un grupo de alrededor de 30 personas, en su mayoría mujeres, se reúne los primeros sábados de cada mes en un bar céntrico de la ciudad de Buenos Aires. Ellas forman parte del club de fans oficial de Ricardo Arjona en la Argentina, un cantante y compositor guatemalteco de gran éxito en la industria discográfica. ${ }^{2}$ Desde allí promocionan su producción musical, realizan tareas solidarias, crean lazos de amistad y compañerismo, así como también elaboran ciclos vitales de manera colectiva. A partir de la apropiación de un objeto musical ellas conforman un grupo de pertenencia y un espacio de socialización complejo al que reconocen como un lugar propio, y afirman que cuando están en las reuniones se sienten "libres" en comparación con 
espacios y vínculos en donde son interpeladas en un cruce de género y ciclo vital que las ubica en el rol de cuidadoras de su hogar y de su familia. El objetivo de este artículo es, entonces, indagar sobre el vínculo entre la música y la configuración de feminidades de este grupo, haciendo especial énfasis en clivajes etarios y generacionales que aparecen en su conformación.

Para ello partimos de un supuesto: en la vida cotidiana las personas interactúan y se apropian de la música de modos en que ésta se constituye en un dispositivo habilitante y promotor de la acción (DENORA, 2000). Con esto queremos decir que entendemos que la música es un recurso que les posibilita reflexionar sobre sí mismas y sus vínculos, auto-representarse, desplegar usos estratégicos para alcanzar 0 modificar ciertas emociones y transitar de un estado anímico indeseado a otro. Asimismo, dentro de este paradigma, haremos foco en la capacidad constitutiva de género que tiene los usos de la música, ${ }^{3}$ específicamente en la configuración de feminidades.

El artículo dará cuenta, en primer lugar, de las discusiones que informan los estudios sobre música y sociedad y, en un segundo momento, indagará sobre el vínculo entre música y mujeres, específicamente sobre el modo en el que este consumo cultural genera habilitaciones respecto de los modos de vivir la posición en la estructura etaria.

\section{Abordajes teóricos para el estudio de la música}

Tres paradigmas informan centralmente los estudios que indagan el vínculo entre música y sociedad: el de resonancia estructural, articulación e interpelación, y narrativa. El primero deriva de los presupuestos de la escuela subculturalista inglesa (HEBDIGE, 2004; CLARKE, 2000; HALL; JEFFERSON, 2000; entre otros) y entiende que ciertos estilos musicales conectarían con actores sociales específicos a

Este artículo forma parte de mi tesis de doctorado para la Facultad de Ciencias Sociales de la Universidad de Buenos Aires, Argentina, titulada ¿Dónde había estado yo?': configuración de feminidades en un club de fans de Ricardo Arjona financiada por becas doctorales del Consejo Nacional de Investigaciones Científicas y Técnicas de la Argentina (CONICET). Asimismo, la investigación que respalda este trabajo forma parte de los proyectos que, con sede la Universidad de Buenos Aires (UBA) y la Universidad de San Martín (UNSAM) y dirigidos por Pablo Alabarces y Pablo Semán, son financiados por UBACYT y el FONCYT.

Edgar Ricardo Arjona Morales, conocido en el ambiente artístico como Ricardo Arjona, es un cantautor nacido en Guatemala en 1964 y de gran éxito en varios países de habla hispana hace más de quince años. Desde su primer disco en 1985 hasta hoy su discografía se compone de trece álbumes de estudio, un álbum en vivo y seis álbumes recopilatorios. Su primer disco lo grabó con la empresa discográfica Discos de Centroamérica (DIDECA), quien posteriormente cedió los derechos a Sony Music de México, compañía para la que trabajará de 1991 hasta 2008. Desde entonces firmó contrato con Warner Music hasta que a fines de 2011 lanzó su productora independiente, Metamorfosis. Su producción hibrida retóricas diversas provenientes del bolero, la balada, la canción de protesta y el pop por lo que no es fácilmente catalogable como perteneciente al mundo de la música romántica, por más que muchas de sus canciones narren historias sobre vínculos erótico-afectivos.

La capacidad constitutiva de género que tiene los usos de la música ya ha sido indagada en diversas investigaciones. Por nombrar sólo algunas: sobre boleros, De la Peza Casares (2001); sobre tango, Savigliano (1995); Archetti (2003); Campodónico y Gil Lozano (2000); Carozzi (2009); Liska (2009); sobre cumbia, Semán y Vila (2006); Silba y Spataro (2008); sobre cuarteto, Blázquez (2008). 
través de una especie de "resonancia estructural" entre posición social por un lado y expresión musical por el otro. Esto es, que la música permitiría expresar o mostrar ciertas identidades construidas previamente. Ahora bien, este tipo de marco teórico puede desembocar en algún tipo de reduccionismo que indicaría una relación de reflejo entre música y sujetos, impidiendo entender de modo más complejo dicho vínculo. El problema consistiría en intentar buscar las conexiones desde la obra musical y los grupos sociales que la consumen o producen, en una relación que sería de homología entre las formas materiales y artísticas. Esto puede observarse en la homologación que suele realizarse tanto entre jóvenes de sectores populares y cumbia como entre personas adultas y tango en la Argentina y, a nivel más general y no sólo en dicho país, entre mujeres y música romántica, por mencionar sólo algunos reduccionismos vinculados a clivajes de clase, etarios y de género respectivamente.

Para sortear el problema de la homología estructural ciertas indagaciones en torno a la música incluyen los conceptos de articulación e interpelación. En esta línea aparecen trabajos como los de Frith (2003) que afirman que el estudio académico de la música popular se vio limitado por el supuesto de que la música debe reflejar o representar de algún modo a las personas, siendo que el problema analítico es intentar rastrear esas huellas entre las obras artísticas y los grupos sociales. La propuesta de Frith es invertir el argumento académico habitual señalando que el problema no es cómo una obra musical refleja a la gente, sino como la misma produce, crea y construye una experiencia identitaria tanto subjetiva como colectiva: su tesis no es que un grupo social tiene creencias que luego refleja en la música, sino que la misma crea esa identidad grupal. Esto es, que los grupos sociales sólo consiguen reconocerse a sí mismo como grupos por medio de la actividad cultural, por medio juicios estéticos como los que habilita, por ejemplo, la música.

Por su parte, Vila (2000) afirma que la teoría de la articulación y las interpelaciones tiene un problema similar a los planteos de la teoría subculturalista inglesa al tener dificultades para explicar por qué una interpelación es más exitosa que otra sin, en última instancia, apelar a algún tipo de homología estructural. El autor afirma que si bien la música no tiene un sentido intrínseco, tampoco sería productivo pensar que el sentido siempre y sólo proviene del oyente: "la música para nosotros sí tiene sentido (no intrínseco, pero sentido al fin), y tal sentido está ligado a las articulaciones en las cuales ha participado en el pasado" (VILA, 2000, p. 14). Llegado a este punto, Vila busca resolver los problemas de la teoría interpelatoria usando los desarrollos de la teoría narrativa introduciendo la idea de trama argumental para pensar los límites a las posibles articulaciones. El autor dirá que la narrativa permite conferirle sentido a las elecciones de los sujetos porque al narrar(nos) otorgamos sentidos y unidad a nuestra vida, ya que la identidad es una identidad narrativa (RICOEUR, 1995), ya sea para el individuo como para la comunidad y es a partir de esta narrativa que habrá cierto abanico de sentidos musicales posibles en detrimento de otros.

Estos tres paradigmas -resonancia estructural, interpelación y narrativa- ayudan a dimensionar 
las discusiones que están en juego en los estudios sobre música: centralmente el vínculo entre causa y efecto de la escucha musical, entre emisión y recepción así como entre música y sociedad. En este sentido, este artículo se alimenta de los hallazgos del campo teórico existente y, asimismo, se propone relocalizar los términos del análisis a partir del trabajo de DeNora (2000), en la medida que el mismo contiene estas discusiones, las condensa y, a la vez, las supera ya que permite poner en cuestión tanto los planteos que entienden la música como reflejo de lo social, así como complejizar los paradigmas que estudian a la música como espacio de configuración de identidades. Así como el trabajo de Frith discute el de los subculturalistas y el de Vila hace lo suyo con el de Frtih, el planteo de DeNora realiza lo mismo con los diferentes paradigmas y pone la discusión en un nuevo punto a desde el cual es posible continuar indagando el vínculo entre música y sociedad y los juegos identitarios que allí se conforman a partir de entender la música como elemento crucial de un dispositivo habilitante y un promotor de la acción. Tal como señaláramos (GARRIGA et. al., 2011), su planteo supera la tensión que podría formularse entre, por ejemplo, el de Adorno (2003) por un lado (y su énfasis en el material musical, en la obra y en el autor) y, por otro, el de Michel de Certeau (1996) (y su énfasis en el uso de la resignificación y la apropiación), ya que allí la música no es meramente un medio "significante" 0 "expresivo" sino que, en el plano de la vida cotidiana, "la música está involucrada en muchas dimensiones del agenciamiento social, en sensaciones, percepciones, en la cognición y conciencia, en la identidad y la energía" tanto individual como colectiva (DENORA, 2000, 16-17). La tesis de la autora podría resumirse de la siguiente manera: en la vida cotidiana las personas interactúan y se apropian de la música de modos en que ésta se constituye en uno de los recursos privilegiados al emprender "la práctica estética reflexiva de subjetivarse a sí mismos y a los otros como agentes emocionales y estéticos a través de los distintos escenarios sociales" (DENORA, 2000, p. 158). Así, la música se vuelve un dispositivo para la autorepresentación, permitiendo a las personas, tal como hemos señalado al comienzo, desplegar "usos estratégicos" para alcanzar o modificar ciertas emociones, salir de un estado anímico indeseado, entre otras habilitaciones.

Tomando como punto de partida este aparato conceptual, en lo que sigue trabajaremos con los relatos de dos mujeres que participan de un club de fans de Ricardo Arjona en la Argentina para indagar qué es lo que la música habilita respecto de los mandatos en torno al género y a la posición en la estructura etaria.

\section{La experiencia musical desde lo etario}

¿De qué modo la música se vincula con el procesamiento social de las edades? ¿Hay ciertas músicas sólo para edades determinadas? La relación entre la música y lo etario es compleja y su estudio suele estar cargado de automatismos: 
parecería que existe música para "jóvenes", para "adultos", música de "viejos", lo que genera los mismos problemas que la etiqueta "música para mujeres". En ambos casos se piensa el vínculo entre música y sociedad desde el paradigma de la resonancia estructural, esto es, que hay músicas que "reflejan" o "expresan" identidades etarias y/o generizadas preexistentes, homogéneas y estancas. A partir de dichas concepciones se esencializan, por un lado, los modos de vivir una posición en la estructura etaria y/o genérica y, por el otro, los posibles vínculos que desde allí pueden armarse en torno a la música.

La producción musical de Arjona es disruptiva respecto de dichas clasificaciones y tematiza lo etario de diversas maneras. Por un lado, con su más famosa canción "Señora de las cuatro décadas", ${ }_{4}^{4}$ que dice

\footnotetext{
Señora de las cuatro décadas/ $Y$ pisadas de fuego al andar/ Su figura ya no es la de los quince/ Pero el tiempo no sabe marchitar/ Ese toque sensual/ $Y$ esa fuerza volcánica de su mirar [...] Señora, no le quite años a su vida/ Póngale vida a los años que es mejor [...] Señora de las cuatro décadas/ № insista en regresar a los 30/ Con sus 40 y tantos encima/ Deja huellas por donde camina/ Que la hacen dueña de cualquier lugar [...].
}

Esta es una de las líricas más significativas para algunas -no todas- de sus seguidoras, quienes encuentran allí la reivindicación de la capacidad de seducción y goce de una mujer que ya no está ubicada en la franja etaria más codiciada en términos eróticos -la juventud- así como de un cuerpo que no corresponde a los cánones de la belleza hegemónica.

Por el otro lado, esta producción musical es disruptiva respecto de lo etario no sólo por dicha lírica sino porque en otras canciones la tematización de las consecuencias del paso del tiempo en la vida de los sujetos, cuerpos y relaciones afectivas es un argumento central. Asimismo, esto es retomado desde los usos de sus fans que, no por ser un objeto cultural catalogado como para "mujeres de mediana edad" por las industrias y los medios, cumplen con ese mandato: a Arjona lo escuchan personas, mayoritariamente mujeres, en una franja etaria amplia que, en el club de fans oficial en Buenos Aires, va de los 14 a los 80 años. En las próximas líneas indagaremos el cruce música, género, edad y generación en los relatos de dos integrantes de dicho grupo. ${ }^{5}$

\subsection{Sobre el sí mismo}

Para estudiar el vínculo entre música y mujeres y, específicamente, el lugar del clivaje etario y generacional en dicha relación, 
analizaremos los relatos de Mirta y de Ana, dos mujeres que crearon el club de fans hace 16 años y hoy forman parte de la comisión directiva del mismo. No preguntaremos cuáles fueron los acontecimientos individuales que las llevaron, en primer lugar, a interesarse por un artista como Arjona; y, en segundo lugar, a armar un club de fans.

Escuché Señora de las cuatro décadas [...] En ese momento yo estaba pasando una situación de vida bastante delicada, mi mamá se quedó ciega (por diabetes) y la tenía conmigo. Y la vi yendo al baño y tenía esa canción en la cabeza y la vi ya grande, que estaba gordita. Entonces después que se acostó ella me miré yo en el espejo. A todo esto te cuento que yo tenía $10 \mathrm{~kg}$ de más y ino estaba embarazada! Entonces me ví y me pregunté cómo había llegado yo a ese punto, que era lo que me había pasado, dónde había estado yo [...] Entonces empecé a hacer dieta, fui al médico, a hacer gimnasia, me puse en órbita y empecé a estudiar italiano. Empecé a hacer cosas para mí, mis hijos eran un poco más grandes [...] Y pensé 'a este tipo hay que escucharlo. Cuando venga a la Argentina lo voy a ir a ver' (Mirta).

Mirta, de 53 años, había pasado su juventud escuchando bandas de rock argentino como Sui generis y Virus, y también a Serrat, dándole en sus elecciones preponderancia a la letra sobre el componente musical y sonoro. Esta predisposición a escuchar las letras de las canciones fue conformando su gusto musical, dato que aparece de manera recurrente en las personas que eligen a este cantautor: ellas prefiere artistas que en sus letras "digan algo" que "les llegue", que cuenten historias, que narren sentimientos de modo que les parezca "claro".

A partir de esta inclinación estética tuvo su primer contacto con Arjona con "Señora de las cuatro décadas". Con esta canción "en la cabeza" Mirta observó a través del espejo los cambios en el cuerpo de su mamá, así como también los del suyo. La canción funcionó aquí como un disparador, como materia prima sobre la que Mirta elaboró su momento vital y el de su madre. Asimismo, en el fragmento de Mirta confluyen otros factores: una transformación que arranca por lo estético -hacer dieta, gimnasia-, lo que le permitiría transitar de otro modo o, por qué no, reinsertarse, en el mercado del deseo y del amor. Por otro lado, darle lugar a actividades que le daban placer, "hacer cosas para ella" porque sus hijos ya estaban más grandes, dando cuenta que las mismas se habían suspendido durante un período en el que "no le daban los tiempos" porque las actividades relacionadas con su maternidad ocupaban gran parte de sus días.

Es allí en donde lo etario necesariamente tiene que ser analizado en su cruce con el género, ya que la pregunta "¿adónde había estado yo?" que se hace Mirta no puede responderse sin ese cruce: había estado cuidando a sus hijos y trabajando, actividades esperables para una mujer de su generación en ese ciclo vital. Es esa trama que allí se teje, y que se pone en cuestión a partir de un relato musical, la que motoriza el vínculo entre 
ella y Arjona. No hay allí una pasividad de escucha que sólo la llevaría a aceptar lo que de ellas diría una canción de su artista favorito. Algo de lo que esta canción dice la "toca" en su subjetividad y le permite, tal como señaláramos con DeNora (2000), reflexionar sobre sí misma y habilitar una pregunta sobre sus deseos, su cuerpo, su tiempo. ${ }^{6}$

Una premisa de este trabajo es que la música que las personas eligen se anuda significativamente con trayectorias vitales particulares a partir de las cuales se le da sentido a esa escucha, trayectorias que están informadas tanto por el clivaje de género como por la pertenencia generacional. Ana y Mirta son parte de una misma generación, pero no por su contemporaneidad cronológica, sino por haber compartido unas mismas condiciones de existencia: son mujeres nacidas en los años sesenta en el seno de familias de clase media de Buenos Aires? ${ }^{7}$ Estas mujeres crecieron en un contexto socio-cultural que históricamente ha definido los roles de las personas en la sociedad de manera dicotómica según el clivaje de género: el amor maternal, el hogar y el cuidado de los hijos fueron parte de la construcción de la feminidad durante la primera mitad del siglo XX (LOBAT0 et. al. 2000), y esta impronta continuó vigente aunque conviviendo con algunos cuestionamientos y modificaciones que comenzaron a tomar forma en la segunda mitad del siglo.

Estas modificaciones tienen que ver con que ellas nacieron en los $60^{\prime}$, una década de cambios en diversos órdenes, sobre todo en la vida cotidiana de las mujeres. La moral sexual y las relaciones entre los género se vieron modificadas, en parte, a partir esos años y ser mujer en los 60 ' fue, como afirma entusiastamente Pujol (2003), distinto a ser mujer apenas unos años antes. Si bien ellas no fueron "mujeres" en los $60^{\prime}$-porque nacieron en ese cambio de década- el contexto sociocultural en el que crecieron comenzó a modificarse a partir de ese momento. Para citar algunos ejemplos: el placer sexual comenzó a escindirse de la reproducción, a partir de una idea de mayor libertad y autoconocimiento, y la píldora anticonceptiva cumplió un importante rol en esta reconfiguración de la moral sexual. Esto significó la redefinición de algunas costumbres, la construcción de nuevas legitimidades y reconfiguración de las relaciones intergenéricas (FEIJO0; DE NARI, 1994). Claro está que estas transformaciones fueron diversas de acuerdo al origen social, cultural y geográfico de los agentes. Centrándonos en las clases medias urbanas -en donde estos cambios tuvieron mayor arraigo (ídem)- a partir de los 60 las

Es este mecanismo uno de los que permite afirmar que el consumo cultural es un momento de producción otro, que no se inscribe en textos sino que tiene que ver con las diversas formas de utilizar los textos que las industrias culturales difunden (DE CERTEAU, 1996; MARTíN BARBER0, 1983).

El concepto de generación, que ha sido desarrollado primero por Mannheim (1993) y después por Bourdieu (1998), indica que para hablar de generaciones no basta la contemporaneidad cronológica; es necesario, además, que se den cambios en las condiciones de existencia que provoquen que los individuos sean generados de una manera distinta; esto es, que actúen y piensen de una manera diferente a los sujetos de otra generación. 
mujeres de ese sector social profundizaron su ingreso al mercado del trabajo y a los estudios universitarios, tensionando al interior de los hogares, entre otras cuestiones, las discusiones sobre quién cuidaba a las/os hijas/os.

Esta convivencia de "lo nuevo" con "lo viejo" respecto del lugar de las mujeres en la sociedad es una característica propia de la década en la que Ana y Mirta nacieron y crecieron. Por ejemplo, Mirta recuerda que quería ser locutora de radio y que su mamá le dijo que no, que tenía que ser algo que fuera independiente, que le diera de comer, pero que le permitiera trabajar desde su casa para poder llevar adelante el hogar y el cuidado de la familia. Por eso estudió odontología, profesión a la que hoy se dedica. Sin embargo, tiene claro que esa no es su vocación, pero que en su época había que hacer lo que decían tus padres, "no te quedaba otra, no es como ahora". En el comentario de la madre de Mirta aparecen combinados los mandatos tradicionales en torno al género así como el cuestionamiento a los mismos: ella le indicó a su hija que debía tener un trabajo para ser "independiente" y, al mismo tiempo, le señaló que el mismo debía realizarse dentro del hogar. De esta manera, nos hallamos en un contexto histórico de rupturas, que señalaban la existencia de ciertos cambios -que Mirta fuera "independiente"-; como continuidades más profundas -pero que trabaje desde su casa-, que ponían en cuestión las concepciones tradicionales con respecto de las conductas esperadas para las mujeres.

Por su parte Ana, de 51 años, otra de las integrantes de la comisión y también fundadora del club, cuenta que siempre le gustó la música y que cuando tenía ocho años sus padres la mandaron a estudiar acordeón a piano porque era un instrumento que por casualidad estaba en su casa. Y si bien ella sentía que era una obligación, reconoce que "era muy obediente, muy cuidadosa de lo que me pedían mis viejos, no era rebelde para nada [...] uno está educado de antes, estás en un casete que te metieron que te cuesta mucho salir de todo eso, no ves otra cosa que eso que te enseñaron". Es por ello que la lírica de Arjona que más le llamó la atención a Ana fue una en la que pudo ver algo distinto a ese mandato familiar:

Encontré [la canción] Jesús verbo y no sustantivo y me pareció maravilloso [...] y la desfachatez de decir... [me impresionaba] la forma sencilla de decirlo y de atreverse a criticar $y$ a todo... a las religiones en general [...] Dice 'me bautizaron cuando tenía 6 meses y a mí no me lo preguntaron'. ¿Por qué te tienen que ubicar en una religión? [...]Y con eso que te decía, que soy tímida, que no me sale... digo: 'uh qué bueno, qué bien que lo dijo' [...] me encantaría tener esa forma de hablar, que uno dice lo que siente y que lo que no, si te gusta bien y sino también, ¿no?" (Ana).

Esta canción ${ }^{8}$ dice

Jesús es más que una simple y llana teoría/ ¿Qué haces hermano leyendo la biblia todo el día?/ Lo que ahí está escrito se resume 
en amor, vamos ve y practícalo [...] Jesús no entiende por qué en el culto le aplauden/ hablan de honestidad sabiendo que el diezmo es un fraude [...] Me bautizaron cuando tenía dos meses y a mí no me avisaron/ hubo fiesta piñata y a mí no me lo preguntaron/ Bautízame tu Jesús por favor, así entre amigos.

Ana encontró allí la manifestación de un "atreverse" a poner en duda los mandatos que vienen como dados, como por ejemplo la religión, y a decir lo que se piensa sin que se interponga la timidez y la autorregulación frente a la opinión del entorno. Encontrar un espacio para cuestionar algunos mandatos familiares es un elemento clave para entender el lugar que estas canciones ocupan en tanto materia prima a partir de la cual balizar las narrativas de sus trayectorias biográficas, así como para reflexionar sobre otros modos posibles de concebir lo social ya no como lo dado sino como una arena posible de ser repensada. Y esta habilitación fue posible, en este caso, a partir del encuentro con una canción que permitió poner en escena un atrevimiento que generó admiración.

\subsection{Crear un club de fans}

Una tarde de 1995 Mirta y Ana, que por ese entonces tenían alrededor 35 años, fueron -al igual que muchas otras personas- al aeropuerto de Ezeiza, aún sin conocerse, con un objetivo: ver personalmente a Arjona. Mirta estaba en un almuerzo con colegas junto a su marido y decidió retirarse antes con el argumento de que tenía que ir al médico. Si bien su marido sabía a dónde iba, a ella le pareció "poco serio" decírselo a sus colegas, ya que podrían poner en cuestión su respetabilidad profesional al conocer su fanatismo. Recuerda que una vez llegada al aeropuerto podían diferenciarse claramente las prácticas de las fanáticas según sus edades:

Yo lo que veía era chicas corriendo y gritando. Entonces, cuando se fueron todos, yo me quedé y Ana estaba ahí, y le dije: 'disculpame, yo te estaba escuchando, a mí me pasa lo mismo que a vos, yo lo descubrí por casualidad en el cable' y empezamos a intercambiar ideas. Le pedí el teléfono y le dije: 'si esto es un club de fans a mí no me gusta esto' y ella me dijo 'a mí tampoco'. Entonces le digo '¿y si le hacemos otro?’ No sé de dónde me salió, te juro que es el día de hoy y lo sigo pensando, ¿de dónde me salió? Y ella me dijo 'no, en mi casa me matan, mi marido me mata'. Yo le dije que me dejara el teléfono $y$ que después nos Ilamáramos a ver si vamos a alguna reunión. Y así empezamos (Mirta).

Es interesante resaltar de este testimonio que ambas deciden armar un club de fans porque los existentes estaban conformados por chicas jóvenes cuyas prácticas no las hacían sentir cómodas.

La figura del fan como un sujeto patológico (JENSEN, 1992), en este caso por la "histeria" que supondrían sus corridas y gritos, está introyectada en el relato de estas mujeres. De esta manera, ellas proponen una lógica de fanatismo que les permite conservar ciertas modalidades que se corresponderían con su lugar de adultas en la estructura etaria, aun en un acto disruptivo para su edad como es armar un club de fans a partir de un gusto intenso por un cantante. Si bien en el mismo ellas van a incorporar a personas jóvenes, lo harán marcando ciertos los límites de lo posible en el grupo: escuchar con atención en lugar de gritar, calmar al desobediente y aprovechar de 
manera inteligente -iadulta?- el tiempo que puedan compartir con Arjona, son los consejos que estas mujeres les dan aquellas personas que quieren formar parte del club de fans. Consejos que están en clara coincidencia con el rol de madres y educadoras para el que han sido formadas.

Asimismo, en el testimonio de Mirta aparece la idea de mujer "madura", que es profesional, madre y esposa, que no coincide, a primera vista, con la figura con la que se relaciona -ni siquiera para ella misma- al término fan. El fanatismo, dirá Lewis (1992), "está asociado con la adolescencia o la niñez, y es representado como femenino, no masculino. La combinación de adolescencia y feminidad en el sistema de representación es significativa" y agrega que "el link ente inmadurez y feminidad opera como una estrategia para burlarse de la mujer y su fanatismo" (LEWIS, 1992, p. 158). Cuando la pretendida coherencia entre edad y práctica se rompe, aparecen los cuestionamientos propios y los del entorno. Y eso se debe a que lo que allí se pone en escena es una incongruencia entre edad y conductas: hay modos de expresar la atracción física e intelectual que un cantante varón puede provocar que no son parte del repertorio esperado de prácticas asociadas a un rango etario y a un género, por lo que ser mujer adulta ubica a la persona fuera de ese juego. Esto debe a que la adultez es un grado de edad, una de las segmentaciones que opera sobre el ciclo vital -al igual que la niñez y la juventud-, que actúa de manera performativa porque, si bien varía históricamente, "los sujetos tienden a adecuarse a la definición social de la categoría [etaria] en la que se hallan definidos" (CRIADO BOADO, 1998, p. 88). Esto quiere decir que hay un sistema de representaciones, estereotipos y valores que legitiman, modelan y performan no sólo los roles de género, sino también los etarios. Y esos roles no sólo están marcados desde afuera, sino que también forman parte de la autopercepción de los sujetos respecto de lo que está bien y lo que está mal, lo que es adecuado e inadecuado para cada grado de edad y ciclo vital. De esta manera, los sujetos aprenden a actuar como adultos y, en el caso de las mujeres, la adultez se configura a partir centralmente de tres lógicas de transición: hacia la profesión, el matrimonio y la maternidad. $\mathrm{Y}$ desde esas posiciones armar o pertenecer a un club de fans supone una ruptura entre edad, género y conductas esperadas, dato que aparece en el relato de Mirta y de Ana, y en el de muchas otras integrantes del grupo:

\section{[...] a mí esto me daba cierta cosita, 'yo, la profesional', nunca me había pasado, a mí me debe estar fallando, tengo dos hijos, soy una mujer casada, encima tenía a mi mamá en mi casa que me decía '¡Vos sos una loca! ¿Cómo vas a ir?'. Pero me fui a Ezeiza (Mirta).}

Cuando empecé yo dije, '¡me matan!' porque imaginate, no es fácil, tengo que hacer esto, salir, ir acá, allá... pero me sentí un poco liberada. Mi marido no sé si tuvo la inteligencia de no decirme 'no, no lo hagas'. Pero protesta, obvio que protesta (Ana).

En ambos casos pueden leerse sus interpretaciones respecto al lugar que ellas ocupan en la estructura etaria y lo que es posible 
de hacer allí, indicando ciertas prácticas como si se tratasen de locuras. Mirta dice que algo le debe estar "fallando". La falla es, claro está, la incoherencia pretendida entre edad, género y conductas: ir a recibir a un ídolo al aeropuerto y armar un club de fans no son prácticas posibles de llevar a cabo por una mujer adulta -profesional y/o madre-. Ese límite fue marcado por sus entornos familiares, así como percibido por ellas mismas.

Pero animarse a dar lugar a esa falla fue vivido también como un modo de cuestionar los límites existentes en sus trayectorias vitales: así como las fans de Madonna producían, a partir de sus elecciones musicales, interpretaciones de su sexualidad que ponían en cuestión los mandatos patriarcales (FISKE, 1989); ${ }^{9}$ las fans de novelas románticas ponían límites a las demandas de su entorno y construían un lugar propio a partir de la lectura de sus textos favoritos (RADWAY, 1991); y las fans de Elvis Presley encontraban en su figura y sus canciones una compañía frente a las angustias y soledades experimentadas en la adolescencia $;{ }^{10}$ la música de Arjona y el club de fans funcionó para sus integrantes, entre otras cosas, como un lugar en donde elaborar colectivamente los sentidos que pueden otorgarse a la propia condición de género y la propia posición en la estructura etaria y, asimismo, una habilitación para vivir de manera más flexible las reglas y fronteras que indicaban los modos en los que debían transitar sus ciclos vital

\section{Conclusiones}

El objetivo de este artículo fue estudiar el vínculo entre música y mujeres y, específicamente, el lugar de las canciones de un cantautor guatemalteco en las configuraciones genéricas y etarias de mujeres que conforman su club de fans. La pregunta que guió el análisis tuvo que ver no tanto con lo que la música de Arjona es y sino con lo que posibilita hacer. Para indagar en ese sentido propusimos corrernos del paradigma de la resonancia estructural ya que la canción "Señora de las cuatro décadas" no "reflejaba" lo que a Mirta le estaba pasando respecto de una relación erótico afectiva con un varón menor que ella ni "Jesús verbo no sustantivo" "expresaba" lo que Ana pensaba respecto de la religiosidad. Al interrogar qué le permite la música hacer a los sujetos advertimos que ambas encontraron en dichas líricas caminos para cuestionar ciertos mandatos aprehendidos en su juventud sobre lo que una mujer podía o no hacer con su tiempo, para tramitar su timidez, para salir de sus casas

Fiske (1992, p. 37) señala que este tipo de prácticas puede ser interpretadas como "productividad semiótica", concepto con el que define la "producción de sentidos de identidad social a partir de los recursos culturales de las mercancías culturales" tanto de la cultura popular en general como en particular de la cultura fan. Para ampliar indagaciones sobre fanatismo ver: Jenkins (1992, 1998, 2001, 2007, 2009); Jensen (1992); Lewis (1992); Harris (1998); Hills (2000); entre otras. Trabajos en la Argentina sobre fans: Borda (2008, 2011); Cragnolini (2001); Martín (2006); Skartveit (2009).

Para ampliar trabajos sobre mujeres y fanatismo Cline (1992); sobre fanáticas de Los Beatles Ehrenreic et. al (1992) y Lewis (1992); sobre fanáticas de Elvis Presley Hinerman (1992). 
así como para reencontrarse con su propio cuerpo.

En definitiva, estas mujeres se autorizaron a cuestionar algunas de sus posiciones desde de una pauta musical que habilitó movimientos en ese sentido.

A partir del uso de aparato conceptual de DeNora (2000) entendemos que la música es parte de un dispositivo que podría llamarse la dimensión estética de la agencia: permite a las personas reflexionar sobre sí mismas y sus vínculos, auto-representarse, hacer inteligibles sus sentimientos, tal como lo hemos señalado. Asimismo, en tanto un recurso habilitante y promotor de la acción, también es un vehículo para construir experiencias que ponen en cuestión las expectativas etarias y de género existentes para mujeres de mediana edad.

\section{Referencias}

ADORNO, Theodor W. Filosofía de la nueva música. Madrid: Akal, 2003.

ARCHETTI, Eduardo. Masculinidades: Fútbol, tango y polo en la Argentina. Buenos Aires: Antropofagia, 2003.

BLÁZQUEZ, Gustavo. Nosotros, vosotros y ellos. Las poéticas de las Masculinidades Heterosexuales entre jóvenes cordobeses. Trans. Revista Transucltural de música Trans. n. 12, 2008. Disponible en: < http:// www.sibetrans.com/trans/trans12/art06.htm >.

BORDA, Libertad. Fan fiction: entre el desvío y el límite. En: ALABARCES, Pablo; RODRÍGUEZ, María Graciela (Comps.) Resistencias y mediaciones: la cultura popular en la Argentina contemporánea. Buenos Aires: Paidós, 2008.

\section{Bettymaniacos, Luzmarianas y}

Mompirris: el fanatismo en los foros de telenovelas latinoamericanas. Tesis (Doctorado en Ciencias de la Sociales) - Facultad de Ciencias Sociales, UBA, 2011.

BOURDIEU, Pierre. La distinción: Criterio bases sociales del gusto. Madrid: Taurus, 1998.

CAMPODÓNIC0, Raúl Horacio; GIL LOZANO.

Milonguitas en-cintas: La mujer, el tango y el cine. En: GIL LOZANO, Fernanda; PITA, Valeria; INI, Gabriela (Ed.). Historia de las mujeres en la Argentina: siglo XX. Buenos Aires: Taurus, 2000.

CAROZZI, María Julia. Una ignorancia sagrada: aprendiendo a no saber bailar tango en Buenos Aires. Religião e Sociedade. v. 29, n. 1, 2009.

CLARKE, John. Style. In: HALL, S.; JEFFERSON, T.

(Ed.) Resistance Through Rituals: Youth Subcultures in Postwar Britain. London; New York: Routledge, 2000.

CLINE, Cherly. Essays from Bitch: The Women's Rock Newsletter with Bite. En: LEWIS, Lisa (Ed.). The Adoring Audience. Fan cultura and popular media. New York: Routeledge, 1992.

COSSE, Isabella. Pareja, sexualidad y familia en los años sesenta. Buenos Aires: Siglo XXI, 2010.

CRAGNOLINI, Alejandra. 'El Ro nos dio tanta vida y se nos fue'. Una aproximación a un cantante de cuarteto a través de las miradas de sus fans. Revista Argentina de musicología, Buenos Aires, n.2, p. 79-94, 2001.

CRIADO BOAD0, Martin. Producir la juventud: crítica de la sociología de la juventud. Madrid: Istmo, 1998.

DE CERTEAU, Michael. La invención de lo cotidiano. México: Universidad Iberoamericana, 1996.

DE LA PEZA CASARES, Carmen. El bolero y la educación sentimental en México. México: UAM-X y Miguel Ángel Porrúa, 2011.

DENORA, Tia. Music in everyday life. New York: Cambridge University Press, 2000. 
EHRENREIC, Barbara; HESS, Elizabeth; JACOBS, Gloria. "Beatlemania: Girls Just Want to Have Fun. En: LEWIS, Lisa (Ed.): The Adoring Audience. Fan cultura and popular media. New York: Routeledge, 1992.

FEIJOÓ, María del Carmen; DE NARI, Marcela. Los 60' de las Mujeres. Todo es historia, n. 321, abr. 1994.

FISKE, John. The Cultural Economy of Fandom. En: LEWIS, Lisa (Comp.) The Adoring Audience. London; New York: Routledge, 1992.

Undestanding Popular Culture. Boston:

Unwin Hyman, 1989.

FRITH, Simon. Música e identidad. En: HALL, Stuart; DU GAY, Paul (Comp.) Cuestiones de identidad cultural. Buenos Aires: Amorrortu, 2003.

GARRIGA, José et al. Introducción. Revista Argentina de Juventud, Observatorio de Jóvenes y Medios de Comunicación de la Facultad de Periodismo y Comunicación Social de la Universidad Nacional de La Plata, n. 4, 2011. Disponible en: < http://www.perio. unlp.edu.ar/revistadejuventud/?q=node/58>

HALL, Stuart; JEFFERSON, Tony (Ed.). Resistencia a tarvés de rituales. Subculturas juveniles en la Gran Bretaña de la posguerra. La Plata: Universidad Nacional de La Plata, Facultad de Periodismo y Comunicación Social, Observatorio de Jóvenes, Comunicación y Medios, 2000.

HARRIS, Cheryl. Introduction. Theorizing Fandom: Fans, Subculture and Identity. En: HARRIS, Cheryl; ALEXANDER, Alison. Theorizing Fandom: Fans, Subculture and Identity. New Yersey: Hampton Press, 1998.

HEBDIGE, Dick. Subcultura: El significado del estilo. Barcelona, Paidós, 2004.

HILLS, Matt. Fan Cultures. New York: Routledge, 2002 .

HINERMAN, Stepehn. I'll Be Here With You: Fans, Fantasy and the Figure of Elvis. En: LEWIS, Lisa (Ed.).
The Adoring Audience. Fan cultura and popular media. New York: Routeledge, 1992.

JENSEN, Joli. Fandom as Pathology: The consequences of characterization. In: LEWIS, Lisa (Ed.). The Adoring Audience. Fan cultura and popular media. New York: Routeledge, 1992.

JENKIS, Henry. Textual Poachers. Television Fans and Participatory Culture. London; New York: Routledge, 1992. The Poachers and the Stormtroopers: Cultural Convergence. In: Digital Age. Charla dictada en la Universidad de Michigan y publicada en Red Rock Eaterews Service. 1998. Disponible en: < http://dlis. gseis.ucla.edu/people/pagre/rre.html> . Intensities interview Henry Jenkins.

Intensities: The Journal of Cult Media, University of Bristol, 7 de julio 2001. Disponible en: < http:// intensities.org/Essays/Jenkins.pdf>. Afterword: The Future of Fandom. En: GRAY, Jonathan;

SANDVOSS, Carl; HARRINGTON, C. Lee. Fandom:

Identities and Communities in a

Mediated World. New York; London: New York University Press, 2007.

Introducción: confesiones de un aca/fan. En:

JENKINS, Henry Fans, blogueros y videojuegos. La cultura de la colaboración, Barcelona: Paidós, 2009.

JENSEN, Joli. Fandom as Pathology: The consequences of characterization. In: LEWIS, Lisa (Ed.) The Adoring Audience. Fan cultura and popular media. New York: Routeledge, 1992.

LEWIS, Lisa. Introduccion. In: . The Adoring Audience. Fan cultura and popular media. New York: Routeledge, 1992.

LISKA, Mercedes. El tango que regula cuerpos ilegítimoslegitimados. Trans, Revista Transcultural de Música, 
n. 13, 2009. Disponible en: <http://www.sibetrans.com/ trans/a53/el-tango-como-disciplinador-de-cuerposilegitimos-legitimados $>$.

LOBATO, Mirta et al. Cuerpos y sexualidad. En: GIL LOZANO, F.; PITA V.; INI, M. G. (Comp.) Historia de las mujeres en la Argentina. Siglo XX. Buenos Aires: Taurus, 2000.

MANNHEIM, Karl. El problema de las generaciones. Revista Española de Investigaciones Sociológicas. Madrid, n. 62, p. 193-242, 1993. Disponible en: < http:// www.reis.cis.es/REISWeb/PDF/REIS_062_12.pdf>

MARTÍN, María Eloisa. No me arrepiento de este amor: Um estudo etnográfico das prácticas de sacralizacao de uma cantora argentina. Tesis (Doctorado en Antropología Social) - Museo Nacional, Universidad Federal de Rio de Janeiro, 2006.

MARTÍN BARBER0, Jesús. Memoria narrativa e industria cultural. Comunicación y cultura, México, n. 10, 1983.

De los medios a las mediaciones. Bogotá: Convenia Andrés Bello, 2003.

PUJOL, Segio. Rebeldes y modernos. Una cultura de los jóvenes. En: JAMES, Daniel (Dir.). Nueva historia Argenitna. Tomo XI. Violencia, proscripción y autoritarismo. Buenos Aires: Sudamericana, 2003.

RADWAY, Janice. Reading the Romance: Women, Patriarchy, and Popular Literature. Chapel Hill; London: University of North Carolina Press, 1991.

RICOEUR, Paul. Tiempo y narración 1. México: Siglo XXI, 1995.

SAVIGLIANO, Marta. Tango and the Political Economy of Passion. Oxford: Westview Press, 1995.

SEMÁN, Pablo; VILA, Pablo. La conflictividad de género en la cumbia villera. Trans, revista transcultural de música, n. 10, 2006. Disponible en: < http://www. sibetrans.com/trans/trans10/vila.htm>
SILBA, Malvina; SPATARO, Carolina. "Cumbia Nena". Jóvenes bailanteras: entre las líricas, los relatos y el baile. En: ALABARCES, Pablo; RODRÍGUEZ, María Graciela (Comp.). Resistencias y mediaciones: Estudios sobre cultura popular. Buenos Aires: Paidós, 2008.

SKARTVEIT, Hanna. Ángles populares: La formación social y espiritual de Gilda y Rodrigo. Buenos Aires: Antropofagia, 2009.

VILA, Pablo. Música e identidad. La capacidad interpeladora y narrativa de los sonidos, las letras y las actuaciones musicales. En: PICCINI, Mabel; MANTECÓN, Ana Rosas, SCHMILCHUK, Graciela (Ed.). Recepción Artística y Consumo Cultural. México: Ediciones Casa Juan Pablos, 2000.

WISE, Sue. Sexing Elvis. En: FRITH, Simon; GOODWIN, Andrew (Ed.) On Record. Rock, Pop, The Written

Word. London: Routeledge, 2006. 
"Lady of the four decades": a study on the link between music, women and age

\section{"Senhora das quatro décadas": um estudo da relação entre música, mulheres e idade}

\section{Abstract}

This paper intends to study the role of music in the configuration of contemporary femininities and, specifically, the age and generational cleavage informing this relationship. First, we will go through three paradigms which inform studies about music and society: structural resonance, interpellation and narrative identity. From this overview, we will relocate the discussion: people appropriate music in such ways that it becomes an enabling and action-oriented device (DeNora, 2000). Second, we will analyze the account of two women who are members of an Argentine fan club devoted to Ricardo Arjona, who has been a highly successful Guatemalan singer and songwriter for over a decade in Spanish-speaking record industry. Our conclusion is that music is an aesthetical resource for agency which enables to erode middle-aged women's age and gender expectations.

\section{Keywords}

Music. Women. Age. Generation.

\section{Resumo}

0 presente trabalho visa interrogar o papel da música na formação das feminidades contemporâneas e, mais especificamente, estudar as clivagens etárias e geracionais presentes no mencionado lazo. Na primeira parte efetuaremos um percurso pelos três paradigmas que informam os estudos sobre música e sociedade: ressonância estrutural, interpelação e identidade narrativa. Contendo os resultados desse percurso relocalizaremos a discussão: as pessoas se apropriam da música de formas que esta torna-se um dispositivo que habilita e promove a acção (DeNora, 2000). Na segunda parte, analisaremos a história de duas mulheres que fazem parte de um fã-clube do Ricardo Arjona, na Argentina. Concluímos que a música é um recurso estético que configura a agência no sentido de redefinir as expectativas habituais sobre a relação entre idade e sexo nas mulheres de meia idade.

\section{Palavras-chave}

Musica. Mulheres. Idade. Geração. 


\section{Expediente}

A revista E-Compós é a publicação científica em formato eletrônico da Associação Nacional dos Programas de Pós-Graduação em Comunicação (Compós). Lançada em 2004, tem como principal finalidade difundir a produção acadêmica de pesquisadores da área de Comunicação, inseridos em instituições do Brasil e do exterior.

\section{E-COMPÓS I www.e-compos.org.br I E-ISSN 1808-2599}

Revista da Associação Nacional dos Programas

de Pós-Graduação em Comunicação.

Brasília, v.15, n.2, maio/ago. 2012.

A identificação das edições, a partir de 2008 ,

passa a ser volume anual com três números.

\section{CONSELHO EDITORIAL}

Afonso Albuquerque, Universidade Federal Fluminense, Brasil Alberto Carlos Augusto Klein, Universidade Estadual de Londrina, Brasil Álvaro Larangeira, Universidade Tuiuti do Paraná, Brasi André Luiz Martins Lemos, Universidade Federal da Bahia, Brasil Ângela Freire Prysthon, Universidade Federal de Pernambuco, Brasil Angela Cristina Salgueiro Marques, Faculdade Cásper Líbero (São Paulo), Brasil Antonio Roberto Chiachiri Filho, Faculdade Cásper Líbero, Brasil Arthur Autran Franco de Sá Neto, Universidade Federal de São Carlos, Brasil Benjamim Picado, Universidade Federal Fluminense, Brasil César Geraldo Guimarães, Universidade Federal de Minas Gerais, Brasil Cristiane Freitas Gutfreind, Pontifícia Universidade Católica do Rio Grande do Sul, Brasil

Denilson Lopes, Universidade Federal do Rio de Janeiro, Brasil Eduardo Peñuela Cañizal, Universidade Paulista, Brasi Eduardo Vicente, Universidade de São Paulo, Brasil Eneus Trindade, Universidade de São Paulo, Brasil Florence Dravet, Universidade Católica de Brasília, Brasil Gelson Santana, Universidade Anhembi/Morumbi, Brasil Gislene da Silva, Universidade Federal de Santa Catarina, Brasil Guillermo Orozco Gómez, Universidad de Guadalajara Gustavo Daudt Fischer, Universidade do Vale do Rio dos Sinos, Brasil Hector Ospina, Universidad de Manizales, Colômbia Herom Vargas, Universidade Municipal de São Caetano do Sul, Brasil Inês Vitorino, Universidade Federal do Ceará, Brasil Jay David Bolter, Georgia Institute of Technology Jeder Silveira Janotti Junior, Universidade Federal de Pernambuco, Brasil John DH Downing, University of Texas at Austin, Estados Unidos José Afonso da Silva Junior, Universidade Federal de Pernambuco, Brasil José Carlos Rodrigues, Pontifícia Universidade Católica do Rio de Janeiro, Brasi José Luiz Aidar Prado, Pontifícia Universidade Católica de São Paulo, Brasil Kelly Cristina de Souza Prudêncio, Universidade Federal do Paraná, Brasil. Laan Mendes Barros, Universidade Metodista de São Paulo, Brasil
Lance Strate, Fordham University, USA, Estados Unidos

Lorraine Leu, University of Bristol, Grã-Bretanha

Lucia Leão, Pontifícia Universidade Católica de São Paulo, Brasil

Malena Segura Contrera, Universidade Paulista, Brasil

Márcio de Vasconcellos Serelle, Pontifícia Universidade Católica de Minas Gerais, Brasil

Maria Aparecida Baccega, Universidade de São Paulo e Escola Superior de Propaganda e Marketing, Brasil

Maria Ataide Malcher, Universidade Federal do Pará, Brasi

Maria das Graças Pinto Coelho, Universidade Federal do Rio Grande do Norte, Brasil

Maria Immacolata Vassallo de Lopes, Universidade de São Paulo, Brasil Maria Luiza Martins de Mendonça, Universidade Federal de Goiás, Brasi Mauro de Souza Ventura, Universidade Estadual Paulista, Brasil Mauro Pereira Porto, Tulane University, Estados Unidos Mirna Feitoza Pereira, Universidade Federal do Amazonas, Brasil Nilda Aparecida Jacks, Universidade Federal do Rio Grande do Sul, Brasil Osvando J. de Morais, Universidade de Sorocaba, Brasil Potiguara Mendes Silveira Jr, Universidade Federal de Juiz de Fora, Brasil Renato Cordeiro Gomes, Pontifícia Universidade Católica do Rio de Janeiro, Brasil Robert K Logan, University of Toronto, Canadá

Ronaldo George Helal, Universidade do Estado do Rio de Janeiro, Brasil Rose Melo Rocha, Escola Superior de Propaganda e Marketing, Brasil Rossana Reguillo, Instituto de Estudos Superiores do Ocidente, Mexico Rousiley Celi Moreira Maia, Universidade Federal de Minas Gerais, Brasil Sebastião Guilherme Albano da Costa, Universidade Federal do Rio Grande do Norte, Brasil

Simone Maria Andrade Pereira de Sá, Universidade Federal Fluminense, Brasil Tiago Quiroga Fausto Neto, Universidade de Brasília, Brasil Suzete Venturelli, Universidade de Brasília, Brasil Valerio Fuenzalida Fernández, Puc-Chile, Chile Veneza Mayora Ronsini, Universidade Federal de Santa Maria, Brasil Vera Regina Veiga França, Universidade Federal de Minas Gerais, Brasil

\section{COMISSÃO EDITORIAL}

Adriana Braga I Pontifícia Universidade Católica do Rio de Janeiro, Brasil

Felipe Costa Trotta I Universidade Federal Fluminense, Brasi

CONSULTORES AD HOC

Adriana Amaral, Universidade do Vale do Rio dos Sinos, Brasil

Ana Carolina Escosteguy, Pontifícia Universidade Católica do Rio Grande do Sul, Brasi Claudia Azevedo, Universidade Federal do Estado do Rio de Janeiro, Brasil Gisela Castro, Escola Superior de Propaganda e Marketing, Brasil Luis Queiroz, Universidade Federal da Paraíba, Brasil Rodrigo Carreiro, Universidade Federal de Pernambuco, Brasil EDIÇÃO DE TEXTO E RESUMOS I Susane Barros SECRETÁRIA EXECUTIVA I Juliana Depiné EDITORAÇÃO ELETRÔNICA I Roka Estúdio TRADUÇÃo I Sieni Campos
COMPÓS I www.compos.org.br

Associação Nacional dos Programas de Pós-Graduação em Comunicação

Presidente

Julio Pinto

Pontifícia Universidade Católica de Minas Gerais, Brasil juliopinto@pucminas.br

Vice-presidente

Itania Maria Mota Gomes

Universidade Federal da Bahia, Brasil

itania@ufba.br

Secretária-Geral

Inês Vitorino

Universidade Federal do Ceará, Brasil

inesvic@gmail.com 